\title{
PERFECT MATRIX METHODS
}

\author{
D. J. FLEMING AND P. G. JESSUP
}

\begin{abstract}
Let $e_{i}=\left(\delta_{i j}\right)_{j=1}^{\infty}, \Delta=\left(e_{i}\right)_{i=1}^{\infty}$ and let $A$ be an infinite matrix which maps $E$ into $E$ where $E$ is an $F K$-space with Schauder basis $\Delta$. Necessary and sufficient conditions in terms of the matrix $A$ are obtained for $E$ to be dense in the summability space $E_{A}$ $=\{x \mid A x \in E\}$ and conditions are obtained to guarantee that $E_{A}$ has Schauder basis $\Delta$. Finally it is shown that if weak and strong sequential convergence coincide in $E$ then in $E_{\boldsymbol{A}}$ the series $\sum_{k} x_{k} e_{k}$ converges to $x$ strongly if and only if it converges to $x$ weakly.
\end{abstract}

1. Introduction. If $x$ is a sequence of scalars and $A=\left(a_{n k}\right)$ is an infinite matrix then by $A x$, the $A$-transform of $x$, we mean the sequence $y$, where $y_{n}=(A x)_{n}=\sum_{k} a_{n k} x_{k}$ provided each of these sums converge. If $E$ is any $F K$-space then $E_{A}$ denotes the collection of all sequences $x$ such that $A x \in E$. The space $E_{A}$ inherits a topology which makes it into an $F K$-space [5, p. 226]. A matrix $A$ with the property that $A x \in E$ whenever $x \in E$ will be called an $E-E$ method. If $A$ is an $E$ - $E$ method then $E \subseteq E_{A}$; if in addition $\bar{E}=E_{A}$ then $A$ is called perfect. Let $\phi$ denote the space of all finitely nonzero sequences, $l$ the space of absolutely summable sequences (with $\|x\|=\sum_{k}\left|x_{k}\right|$ ) and $\Delta=\left(e_{i}\right)_{i=1}^{\infty}$, where $e_{i}$ is the sequence $\left(\delta_{i j}\right)_{j=1}^{\infty}$.

In [3] it is shown that a reversible $l-l$ method is perfect if and only if the matrix $A$ has no nonzero left annihilators in $m$, the space of bounded sequences. In [2] conditions are obtained for a general $l-l$ method to be perfect. It is also shown in [2] that the series $\sum_{k} x_{k} e_{k}$ converges strongly to $x \in l_{A}$ if and only if it converges weakly to $x$. The purpose of this note is to show that many of the results obtained in [2] and [3] for the summability field of an $l-l$ method carry over to the summability field of an $E-E$ method when $E$ is an $F K$-space with basis $\Delta$. In particular we show (Theorem 9) that if weak and strong sequential convergence coincide in $E$ then for $x \in E_{\Delta}$ the series $\sum_{k} x_{k} e_{k}$ converges to $x$ strongly if and only if it converges weakly and (Theorem 2) that a reversible $E-E$ method $A$ is perfect if and only if $A$ has no nonzero left annihilators in the sequence space representation of its dual. We will assume throughout this note that $E$

Received by the editors July 1, 1970 .

AMS 1969 subject classifications. Primary 4031, 4046.

Key words and phrases. Perfect matrix method, associative matrix method, property $\mathrm{P}$, type $M$, type $M^{*}$. 
is an $F K$-space with basis $\Delta$ and so in particular every such space contains $\phi$.

2. Notation and terminology. An $E-E$ method is said to be reversiible if the equation $y=A x$ has a unique solution $x$ for each $y \in E$. If $A$ is a reversible $E-E$ method then $E_{A}$ is topologically isomorphic to $E$ under the map $A$ [5, Corollary 5, p. 204, Corollary 1, p. 199]. If the $E-E$ method $A$ is reversible then every $f \in E_{A}^{*}$ can be written in the form $g \circ A$ for $g \in E^{*}$, where ${ }^{*}$ denotes the space of continuous linear functionals.

If $x$ and $y$ are sequences then $(x, y)$ will denote the sum $\sum_{k} x_{k} y_{k}$ and $x A$ denotes the sequence $\left(\sum_{n} x_{n} a_{n k}\right)_{\mathbf{k}=1}^{\infty}$. For $E$ an $F K$-space let $E^{\delta}=\left\{t_{f} \mid f \in E^{*}\right\}$, where $t_{f}=\left(f\left(e_{n}\right)\right)_{n=1}^{\infty}$. Let $b s$ denote the set of sequences with finite norm $\|x\|=\sup _{n}\left|\sum_{j=1}^{n} x_{j}\right|$, cs the set of sequences $x$ for which $\sum_{k} x_{k}$ converges with the norm inherited from $b s, b v$ the space of sequences of bounded variation with $\|x\|=\left|x_{1}\right|+$ $\sum_{k}\left|x_{k}-x_{k+1}\right|, c_{0}$ the sequences which converge to zero with the sup norm and $b v_{0}=b v \cap c_{0}$ with the norm of $b v$. Each of the above is a $B K$-space. Finally we let $\omega$ denote the $F K$-space of all scalar sequences with the product topology.

3. Principal results. Motivated by the notions of type $M$, type $M^{*}$ (see, for example, $[1$, p. 90], [4, p. 184] and [2, p. 358]) and the fact that $l^{\delta}=m$ and $c^{\delta}=l$ we make the following definition.

Definition 1. An $E-E$ method $A$ is said to be of type $E^{\delta}$ if whenever $t A=0$ for $t \in E^{\delta}$ then $t=0$.

Theorem 2. Let $A$ be a reversible $E-E$ method; then $A$ is perfect if and only if $A$ is of type $E^{\delta}$.

Proof. $(\Leftarrow)$ It suffices to show that $\Delta$ is a fundamental set in $E_{A}$. Let $f \in E_{A}^{*}$ and suppose that $f\left(e_{k}\right)=0$ for each $k$. Since $f \in E_{A}^{*}$ there exists a $g \in E^{*}$ with $f=g \circ A$. Thus $0=f\left(e_{k}\right)=g\left[A e_{k}\right]=g\left[\left(a_{1 k}, a_{2 k}, \cdots\right)\right]$ for each $k$. For $g \in E^{*}$ and $x \in E, g(x)=\sum_{n} g\left(e_{n}\right) x_{n}$ and hence $\sum_{n} g\left(e_{n}\right) a_{n k}=0$ for each $k$. Since $A$ is of type $E^{\delta}$ it follows that $g\left(e_{n}\right)=0$ for each $n$ and hence $g \equiv 0$. Thus for $x \in E_{A}, f(x)=g[A x]=0$ and so $\Delta$ is a fundamental set in $E_{A}$.

$\Leftrightarrow$ Assume now that $\bar{E}=E_{A}$ and that $t_{f} A=0$ for some $f \in E^{*}$. Let $F_{a}$ denote the $E_{A}$ topology and let $A \mid E$ denote $A$ considered as a linear operator from $E$ into $E$. Since $A: E_{A} \rightarrow E$ is continuous and $f \in E^{*}$ it follows that $f \circ A \mid E \in\left(E, F_{a}\right)^{*}$. Furthermore $\Delta$ is a basis for $\left(E, F_{a}\right)$ since the $F_{a}$ topology is weaker than the topology of $E \quad\left[5\right.$, p. 203]. Now $f\left[A e_{k}\right]=f\left[\sum_{n} a_{n k} e_{n}\right]=\sum_{n} a_{n k} f\left(e_{n}\right)=\left(t_{f} A\right)_{k}$. 
Therefore $\phi \subseteq(f \circ A \mid E)^{\perp}$ but $(f \circ A \mid E)^{\perp}$ is $F_{a}$-closed in $E$ and $\phi$ is $F_{a}$-fundamental in $E$, hence $f \circ A \mid E \equiv 0$. The zero functional and $f \circ A$ are both continuous extensions of $f \circ A \mid E$ to all of $E_{A}$. Since $\bar{E}=E_{A}$ it follows that $f \circ A \equiv 0$ and hence by the reversibility of $A$, $f \equiv 0$. Thus $t_{f}=0$ and $A$ is of type $E^{f}$.

Since $l^{\delta}=m$ we obtain as a corollary the following theorem of Brown and Cowling [3, Theorem 2].

COROLlary 3. A reversible l-l method is perfect if and only if it is of type $M^{*}$.

Similarly for reversible $E$ - $E$ methods, where $E$ is one of the familiar sequence spaces $c s, c_{0}$ or $b v_{0}$, we have that perfectness is equivalent to type $b v$, type $l$, and type $b s$ respectively.

Definition 4. If $A$ is an $E$ - $E$ method and $t \in E^{\delta}$ we say that $t$ has property $\mathrm{P}$ if $(t A, x)=\sum_{k} \sum_{n} t_{n} a_{n k} x_{k}$ converges for each $x \in E_{A}$. The set of all $t \in E^{\delta}$ with property $\mathrm{P}$ is denoted by $Q$. The method is called associative if $Q=E^{\delta}$ and $f[A x]=\left(t_{f} A, x\right)$ for each $f \in E^{*}$ and each $x \in E_{A}$ (cf. [2, p. 282]).

Lemma 5. Let $A$ be an $E-E$ method and let $t \in Q$ then $(t A, \cdot)$ defines a continuous linear functional on $E_{A}$.

Proof. Let $g_{j}=\sum_{k=1}^{J}\left(\sum_{n} t_{n} a_{n k}\right) E_{k}$ and $g(x)=(t A, x)$, where $E_{k}$ is the $k$ th coordinate functional. Since $E_{A}$ is an $F K$-space $g_{j} \in E_{A}^{*}$ for each $j$ and since $t \in Q, g_{j} \rightarrow g$ pointwise on $E_{A}$. The continuity of $g$ follows from [5, p. 200].

THEOREM 6. Let $A$ be an E-E method. Then $A$ is perfect if and only if $f[A x]=\left(t_{f} A, x\right)$ for each $x \in E_{A}$ and each $t_{f} \in Q$ (cf. [3, Theorem 1] and $[2$, Theorem $\mathrm{A}])$.

Proof. $(\Rightarrow)$ Let $t_{f} \in Q$ and let $g(x)=\left(t_{f} A, x\right)$ for $x \in E_{A}$; then $f\left[A e_{j}\right]=\sum_{i} a_{i j} f\left(e_{j}\right)=\left(t_{f} A, e_{j}\right)$ and so $g=f \circ A$ on the fundamental set $\Delta$. Since $g$ and $f \circ A$ are continuous on $E_{A}$ it follows that $g \equiv f \circ A$.

$(\Leftrightarrow)$ Let $f \in E_{A}^{*}$ be such that $f\left(e_{k}\right)=0$ for each $k$. By [5, p. 230] there exists $F \in \omega_{A}^{*}$ and $G \in E^{*}$ such that $f(x)=F(x)+G[A x]$ for each $x \in E_{A}$. Therefore $0=f\left(e_{k}\right)=F\left(e_{k}\right)+G\left[\sum_{n} a_{n k} e_{n}\right]=F\left(e_{k}\right)+\sum_{n} a_{n k} G\left(e_{n}\right)$. Since $\Delta$ is a basis for $\omega_{A}[5$, p. 230] we have in particular that $F(x)$ $=\sum_{k} F\left(e_{k}\right) x_{k}$ for each $x \in E_{A}$. Combining these results we have that

$$
\sum_{k} F\left(e_{k}\right) x_{k}=-\sum_{k}\left(\sum_{n} G\left(e_{n}\right) a_{n k}\right) x_{k}
$$

for each $x \in E_{A}$. Thus 


$$
\begin{aligned}
f(x) & =F(x)+G[A x] \\
& =\sum_{k} F\left(e_{k}\right) x_{k}+\sum_{n} G\left(e_{n}\right) \sum_{k} a_{n k} x_{k} \\
& =\sum_{n} G\left(e_{n}\right) \sum_{k} a_{n k} x_{k}-\sum_{k}\left(\sum_{n} G\left(e_{n}\right) a_{n k}\right) x_{k} \\
& =G[A x]-\left(t_{f} A, x\right)=0 .
\end{aligned}
$$

Hence $f \equiv 0$ and so $\bar{E}=E_{A}$.

Theorem 7. Let $A$ be an $E-E$ method. Then $A$ is associative if and only if $E_{A}$ has basis $\Delta$.

Proof. $(\Rightarrow)$ Let $x \in E_{A}$ and $f \in E_{A}^{*}$. Choose $F \in \omega_{A}^{*}, G \in E^{*}$ such that $f=F+G \circ A$ and let $y_{n}=x-\sum_{k=1}^{n-1} x_{k} e_{k}$. Then

$$
\begin{aligned}
f\left(y_{n}\right) & =F\left(y_{n}\right)+G\left[A y_{n}\right]=F\left(y_{n}\right)+\left(t_{G} A, y_{n}\right) \\
& =F\left(y_{n}\right)+\sum_{k=n}^{\infty}\left(\sum_{j=1}^{\infty} G\left(e_{j}\right) a_{j k}\right) x_{k} .
\end{aligned}
$$

The first term limi? s to 0 on $n$ since $\Delta$ is a basis for $\omega_{A}$ and the second limits to 0 since the double series converges. Thus $\Delta$ is a weak basis and hence a basis for $E_{A}$.

$(\Leftarrow)$ Let $x \in E_{A}$ and $f \in E^{*}$ then $f \circ A \in E_{A}^{*}$ and so

$$
f[A x]=\sum_{k} x_{k} f\left[A e_{k}\right]=\sum_{k} x_{k} \sum_{n} a_{n k} f\left(e_{n}\right)=\left(t_{f} A, x\right) .
$$

We shall say that $x \in E_{A}$ is perfect if $f(A x)=\left(t_{f} A, x\right)$ for each $t_{f} \in Q$ and that $x$ is associative if $Q=E^{\delta}$ and $f(A x)=\left(t_{f} A, x\right)$ for all $t_{f} \in Q$.

TheOREM 8. Let $A$ be an $E-E$ method and let $x \in E_{A}$; then

(i) $\sum_{k} x_{k} e_{k}$ converges to $x$ weakly if and only if $x$ is associative,

(ii) $x$ is in the closure of $\phi$ in $E_{A}$ if and only if $x$ is perfect.

Proof. (i) $(\Rightarrow)$ Let $t_{f} \in E^{\delta}$ and let $F=f \circ A$; then $F \in E_{A}^{*}$ and $F(x)=\sum_{k} x_{k} F\left(e_{k}\right)=\sum_{k} x_{k} f\left(A e_{k}\right)=\sum_{k} x_{k} \sum_{n} a_{n k} f\left(e_{n}\right)=\left(t_{f} A, x\right)$.

$(\Leftarrow)$ Let $g \in E_{A}^{*}$; say $g=F+G \circ A$ for $F \in \omega_{A}^{*}$ and $G \in E^{*}$; then $g\left(e_{k}\right)=F\left(e_{k}\right)+\sum_{n} G\left(e_{n}\right) a_{n k}$. Thus

$$
\begin{aligned}
g(x) & =F(x)+G[A x]=\sum_{k} x_{k} F\left(e_{k}\right)+G[A x] \\
& =\sum_{k} x_{k}\left(g\left(e_{k}\right)-\sum_{n} G\left(e_{n}\right) a_{n k}\right)+G[A x] \\
& =\sum_{k} g\left(e_{k}\right)-\left(t_{G} A, x\right)+G[A x]=\sum_{k} x_{k} g\left(e_{k}\right) .
\end{aligned}
$$


(ii) $(\Rightarrow)$ Let $x$ be in the closure of $\phi$ in $E_{A}$ and let $t_{f} \in Q$. Define $g: E_{A} \rightarrow k$ by $g(y)=f[A y]-\left(t_{f} A, y\right)$; then $g \in E_{A}^{*}$ by Lemma 5 but $g\left(e_{k}\right)=0$ for each $k$ and so $g(x)=0$. Therefore $f[A x]=\left(t_{f} A, x\right)$.

$(\Leftarrow)$ Let $f \in E_{A}^{*}$ be such that $f \mid \phi \equiv 0$. Then, as in (i), $f(x)=\sum_{k} f\left(e_{k}\right) x_{k}$ $+G[A x]-\left(t_{G} A, x\right)=G[A x]-\left(t_{G} A, x\right)$. Thus $t_{G} \in Q$ and so $f(x)=0$.

For the following theorem we do not assume $E$ has basis $\Delta$.

TheOREM 9. Let $A$ be an E-E method and suppose that weak and strong sequential convergence coincide in $E$. Then for $x \in E_{A}$ the series $\sum_{k} x_{k} e_{k}$ converges to $x$ if and only if it converges to $x$ weakly.

PRoof. Let $x \in E_{A}$ be such that $\sum_{k=1}^{n} x_{k} e_{k} \rightarrow x$ weakly and let $y_{j}=\left(0, \cdots, 0, x_{j}, x_{j+1}, \cdots\right)$. Let $\left(r_{n}\right)$ be the determining seminorms for $E$; then the topology of $E_{A}$ is given by the seminorms $\left(\left|E_{n}\right|\right)$, $\left(p_{n}\right),\left(q_{n}\right)$, where $q_{n}=r_{n} \circ A$ and $p_{n}$ is defined by

$$
p_{n}(x)=\sup _{m}\left|\sum_{k=1}^{m} a_{n k} x_{k}\right| \quad[5, \text { p. 226, Theorem 1]. }
$$

Since $E_{n} \in E_{A}^{*}$ for each $n$ it is clear that $\left|E_{n}\left(y_{j}\right)\right| \rightarrow_{j} 0$ for each $n$. Let $f \in E^{*}$ then $f \circ A \in E_{A}^{*}$ and hence $f \circ A\left(y_{j}\right) \rightarrow_{j} 0$. Thus $\left(A\left(y_{j}\right)\right)$ converges to zero weakly and hence strongly in $E$ and so $q_{n}\left(y_{j}\right) \rightarrow 0$ for each $n$. Finally fix $n$ and let $\epsilon>0$ be given. Choose $N$ such that $j, m \geqq N$ implies

$$
\left|\sum_{k=j}^{m} a_{n k} x_{k}\right|<\epsilon
$$

Thus

$$
\sup _{m>j}\left|\sum_{k=j}^{m} a_{n k} x_{k}\right| \leqq \epsilon \quad \text { for } j>N,
$$

but $p_{n}\left(y_{j}\right)=\sup _{m}\left|\sum_{k=j}^{m} a_{n k} x_{k}\right|$ and hence $p_{n}\left(y_{j}\right) \rightarrow_{j} 0$ for each $n$. It follows that $y \rightarrow_{j} 0$ in $E_{A}$.

REMARKs. (i) It has been pointed out by G. Bennett that the proof of Lemma 3 on p. 285 of [2] makes incorrect use of Satz 3.4 of [6, p. $60]$. Since weak and strong sequential convergence coincide in $l$ Lemma 3 of [2] follows from Theorem 9 above.

(ii) If $E$ is an $F K$-space with determining seminorms $\left(r_{n}\right)$ and if $A$ is a row finite $E-E$ method then the seminorms $\left(\left|E_{n}\right|\right)$ and $\left(r_{n} \circ A\right)$ are sufficient to determine the topology of $E_{A}$. Thus if weak and strong sequential convergence coincide in $E$ one can proceed as in the proof of Theorem 9 to show they coincide in $E_{A}$. This result has been observed by Bennett in [7]. 


\section{REFERENCES}

1. S. Banach, Théorie des opérations linéaires, Monografie Mat., PWN, Warsaw, 1932; reprint, Chelsea, New York, 1955. MR 17, 175.

2. H. I. Brown, The summability field of a perfect ll method of summation, J. Analyse Math. 20 (1967), 281-287. MR 36 \#1869.

3. H. I. Brown and V. F. Cowling, On consistency of $l-l$ methods of summation, Michigan Math. J. 12 (1965), 357-362. MR 32 \#1480.

4. M. S. Ramanujan, On summability methods of type $M$, J. London Math. Soc. 29 (1954), 184-189. MR 15, 697.

5. A. Wilansky, Functional analysis, Blaisdell, New York, 1964. MR 30 \#425.

6. K. Zeller, Abschnittskonvergenz in FK-Räumen, Math. Z. 55 (1951), 55-70. MR 13, 934.

7. G. Bennett, $A$ representation theorem for summability domains, Pacific J. Math. (to appear).

Clarkson College of Technology, Potsdam, New York 13676 